\title{
dispositions intéressant le sol et les fondations dans les nouvelles règles parasismiques P.S. 86
}

\author{
ground conditions and foundations requirements \\ in the new French code \\ of earthquake resistant building design (P.S. 86)
}

\author{
J.F. CORTE \\ Chef de la Division Géotechnique - Mécanique des Sols* \\ A. ISNARD \\ Chef de la Division Sols et Fondations du Bureau Veritas * *
}

\section{Résumé}

Les règles parasismiques dites «P.S. 69 " présentent, tant à l'égard du comportement des sols soumis à l'action sismique qu'à celui de la conception et du dimensionnement des fondations un certain nombre de lacunes auxquelles se trouve confronté le projeteur. Les nouvelles règles dites provisoirement P.S. 86 , en intégrant les connaissances récemment acquises dans ce domaine, différencient davantage les mouvements sismiques en fonction du contexte géotechnique et de la topographie des lieux.

Outre des dispositions constructives détaillées, elles présentent des critères d'identification des sols dits "liquéfiables " et des méthodes pratiques de vèrification de la stabilité des organes de fondation en interaction dynamique avec le sol et les structures portées.

\footnotetext{
Abstract

Civil Eneineers have to face some lack of information concerning the soils behaviour prediction and the design of foundations when using the P.S. 69 French Code of Earthquake Resistant Building Design.

The noticeable amount of observations, pratical and theorical knowledge acquired since this Code publication justifies now, in the new P.S. 86 Code, to differentiate the seismic movement elastic spectra according to geotechnical and soil surface actual conditions This Code, which provides detailed construction requirements, offers also pratical methods for liquefaction risk prediction and for foundation design, taking in account soil-structure interaction under seismic action.
} 


\section{INTRODUCTION}

L'étude de l'effet des séismes sur le comportement des terrains, des fondations et celle des phénornènes d'interaction entre sol et structures ont pris une place croissante dans les travaux de recherche en mécaniques des sols à partir du milieu des années 60. L'étendue des dégâts occasionnés la même année en 1964 par deux tremblements de terre au Japon à Niigata et en Alaska a été un élément initiateur prépondérant. Depuis lors, les connaissances acquises ont été considérables, et, s'il demeure de nombreuses incertitudes ou si les méthodes d'analyse restent largement imparfaites, l'ingénieur peut actuellement intégrer bon nom. bre des résultats de ces recherches en vue d'améliorer la sécurité des constructions en zone sismique.

Le contenu d'un texte réglementaire est toujours le reflet des connaissances au moment de sa rédaction. A cet égard, les règles dites P.S. 69 illustrent bien les lacunes importantes auxquelles se trouvait en butte le projeteur dans les années soixante pour appréhender l'influence des conditions géotechniques locales et justifier en particulier le bon comportement des fondations.

Les nouvelles règles P.S. 86 font cette fois une place importante aux aspects géotechniques, ce qui, somme toute, paraît naturel dans la mesure où d'une part l'action sismique s'impose à la structure par le biais du sol de fondation et qu'une large proportion des sinistres a été imputable à une défaillance du sol d'assise ou des fondations.

Le présent article passe en revue les différentes dispositions intéressant le sol et les fondations introduites dans les nouvelles règles P.S. 86 en apportant quelques commentaires sur les raisons de certains choix, dont tous ne peuvent pas être rigoureusement établis. Sur les différents points évoqués, on notera l'évolution par rapport aux dispositions contenues dans les règles P.S. 69

\section{RÈGLES GÉNÉRALES POUR LA CONCEPTION DES PROJETS}

\subsection{Le choix du site}

Les règles P.S. 86 traitent explicitement trois situations particulières pour lesquelles des contraintes de construction sont fixées.

\section{Le voisinage des failles actives}

Les informations sur la nature et l'intensité du mouvement du sol au voisinage immédiat d'une faille rejouant au cours d'un séisme sont pratiquement inexistantes. Il est tenu pour peu probable que les règles de construction préconisées en champ lointain soient également efficaces dans une telle zone. Ceci a conduit d'une part à exclure toute construction dans une bande de l'ordre de $50 \mathrm{~m}$ de large de part et d'autre d'une faille tenue pour active, et d'autre part à majorer le mouvement sismique de calcul pour les ouvrages implantés à moins de $300 \mathrm{~m}$ de la faille.

\section{La stabilité des pentes et talus}

Aucun ouvrage ne doit être édifié sur un site directement menacé par l'éboulement ou le glissement des fonds supérieurs. Si un tel régime n'est pas déjà identifié sur un document cartographique (PER, ZERMOS, POS, ...), il sera nécessaire de s'assurer de la stabilité d'ensemble du site.

\section{Zones suspectes de liquéfaction}

Si la présence d'une zone liquêfiable n'implique pas nécessairement l'abandon du site, la construction ne peut cependant être entreprise que s'il est établi que la liquéfaction ne représente aucun danger pour l'ouvrage ou si le sol a subi un traitement dont il peut être prouvé qu'il élimine le danger de liquéfaction.

Le phénomène de liquéfaction des sols, simplement évoqué dans les règles P.S. 69 , est maintenant une préoccupation importante et les règles P.S. 86 font une large place aux justifications à appporter vis-à-vis de ce risque.

\subsection{Les reconnaissances et études de sol}

Par rapport aux situations non sismiques, les investigations géotechniques doivent ici, dans tout projet, répondre à deux objectifs

- permettre le classement du site par rapport aux sites types en vue de définir le mouvement sismique de calcul,

- détecter les formations a priori suspectes de liqué. faction,

Ces informations peuvent être obtenues par des méthodes traditionnelles : essais d'identification, essais en laboratoire et en place. Par contre, des études complémentaires spécifiques sont nécessaires :

- en présence d'une zone suspecte de liquéfaction.

- lorsque l'on désire utiliser une méthode de calcul impliquant la prise en compte des propriétés géomécaniques des sols sous chargement dynamique.

\subsection{L'implatation de l'ouvrage et la conception du mode de fondation}

Les recommandations faites sur ces questions étaient pour l'essentiel déjà contenues dans les règles P.S. 69 ; elles procèdent du bon sens :

- choisir, dans la mesure du possible, des formations homogènes et compactes,

- implanter l'ouvrage tout entier d'un même côté de discontinuités géologiques.

- exclure les sols pouvant donner lieu à des effondrements de structure (cinérites, loess, ...) ou à des fortes réductions de l'indice des vides (remblais insuffisarnment compactés)

- encastrer la construction dans le sol et, si plusieurs solutions correspondant à des niveaux d'assise différents peuvent être envisagés, préférer la solution la plus profonde. 
- définir un système de fondation homogène,

- liaisonner les éléments de fondation (semelles ou fondations profondes) entre eux pour s'opposer aux déplacements relatifs.

\section{INCIDENCE DES CONDITIONS GÉOTECHNIQUES LOCALES}

\section{SUR LA DÉFINITION}

\section{DU MOUVEMENT SISMIQUE DE CALCUL}

\subsection{La situation dans les règles P.S. 69}

Dans les règles P.S. 69 , la nature du sol de fondation intervenait à deux reprises pour modifier la valeur du coefficient sismique $\sigma$ applicable à la construction considérée, cela, à travers le coefficient de réponse $\beta$ et le coefficient de fondation $\delta$.

Étant reconnu que les formations épaisses de terrains meubles se comportent comme des filtres amortissant quelque peu les composantes hautes fréquences de l'ébranlement sismique, les spectres de calcul $\beta(T)$ étaient écrêtés d'environ $15 \%$ pour les structures ayant une courte période propre (inférieure à $0,5 \mathrm{~s}$ ) fondées sur de tels sites.

Si les sols meubles donnent ainsi dans certaines situations, par atténuation, un effet favorable par rapport aux terrains rocheux, par contre, à sollicitations sismi- ques égales, les risques de déplacements différentiels sont à l'évidence plus importants pour des constructions fondées sur sol meuble. C'est pour tenir compte de ce fait que le coefficient sismique $\sigma$ était pondéré par un facteur $\delta$ dont la valeur dépendait à la fois de la nature du sol et de celle du système de fondation.

Si cette approche recoupe effectivement certains aspects de l'influence des conditions géotechniques locales, elle présente quelques insuffisances importantes auxquelles les règles P.S. 86 tentent de remédier. Nous énumérons d'abord ces lacunes avant d'examiner la manière dont ces questions sont traitées dans les nouvelles règles

- Si les spectres de réponse élastique pour les mativements enregistrés en surface de terrains meubles montrent une atténuation par rapport aux mouve ments obtenus sur affleurement rocheux pour les cour. tes périodes, par contre, ils doivent présenter une amplification pour les périodes plus élevées. Ces deux tendances associées à un décalage vers les basses fréquences de la période fondamentale de réponse du site lui-même sont maintenant nettement établies par dé multiples enregistrements et des analyses statistiques de distribution de dommages. Les spectres de calcul doivent donc tenir compte de cette amplification par quasi-résonance du mouvement des constructions souples établies sur terrain meuble (fig. 1).

- Pour obtenir des niveaux de protection comparables selon les sites, il faut encore différencier les conditions de site.

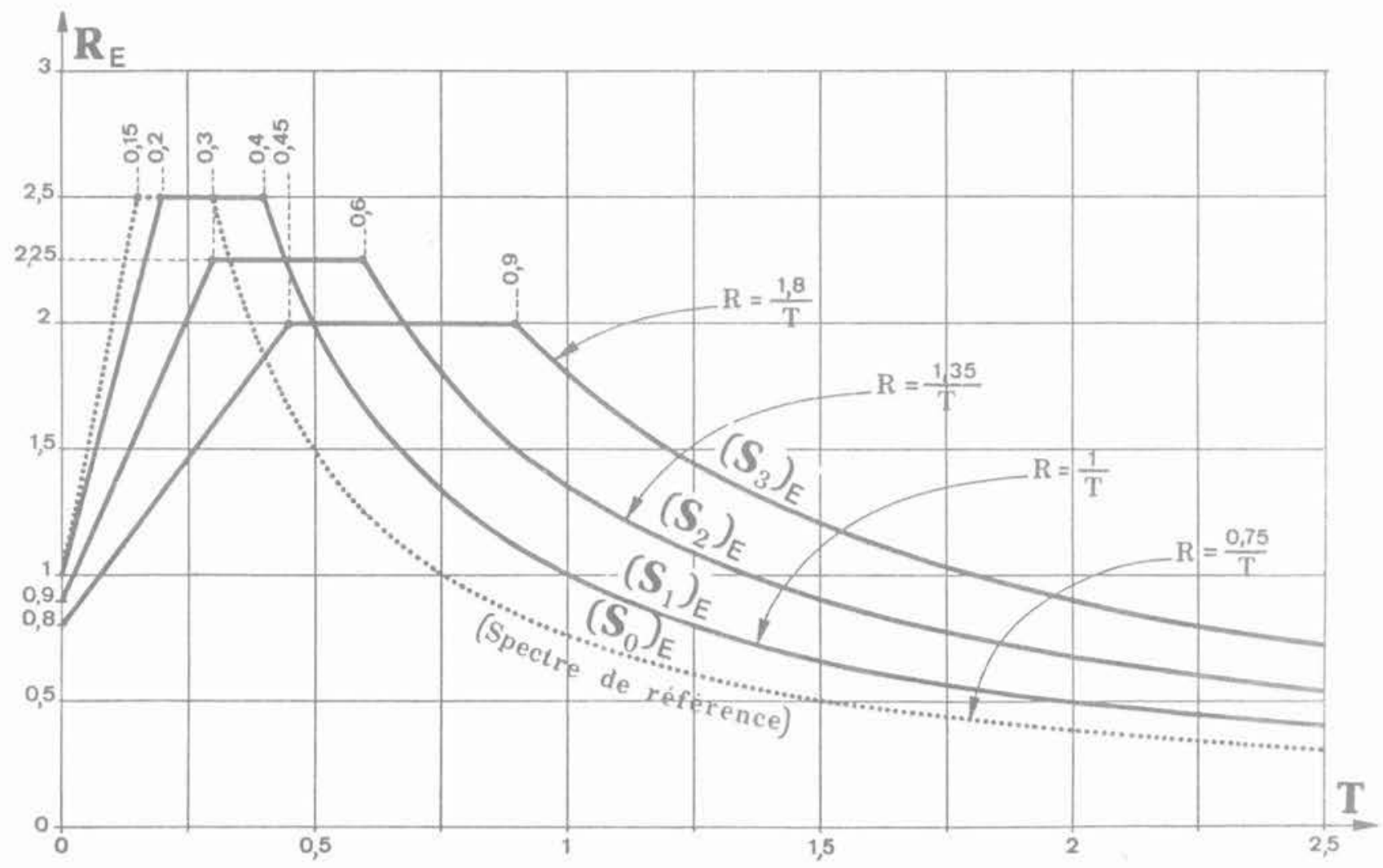

Fig. 1. 
- Les critères géomécaniques d'identification et de classement des sols sont insuffisants.

- Le coefficient $\delta$ apparaît comme trop arbitraire et trop forfaitaire pour traiter correctement à la fois de l'influence du mode de fondation sur le comportement de la construction et des risques de tassements différentiels.

\subsection{Différenciation des spectres élastiques normalisés selon les sites}

Le mouvement sismique que l'on obtient en surface en champ libre dépend des propriétés géomécaniques des formations géologiques du site et de la topographie. $\mathrm{Ce}$ second facteur d'influence est traité par un coefficient correcteur $\tau$, présenté plus loin. Pour une zone sans relief, on définit alors, à partir d'une double classification des sols et des sites, quatre spectres élastiques normalisés.

Outre le rocher sain et les craies dures, les terrains sont regroupés en trois catégories (fig. 2), à partir des résulltats d'essais géomécaniques. Les sites sont eux-mêmes classés en quatre types selon la nature des sols et l'épaisseur des couches (fig. 3).

Il importe encore de noter que dans le cas de sites comportant des sols du groupe $C$ en très grande épaisseur (plus de $100 \mathrm{~m}$ ). il convient de procéder à une étude particulière pour déterminer un spectre approprié. Une telle situation conduisant généralement à un pic d'amplification très marqué au voisinage du premier mode de vibration du dépôt, ne peut pas en effet être traitée correctement par la donnée de spectres moyens.

\subsection{L'influence de la topographie}

Si les constatations post-séisme montrent en général un accroissement sensible des dommages occasionnés aux constructions édifiées sur une pente ou au voisinage d'une crête de talus, les données et modèles disponibles pour quantifier précisément ces effets sont encore quasi inexistants. Pour ne pas cependant ignorer cette question, les règles P.S. 86 proposent de modifier globalement la valeur du mouvement sismique par un coefficient multiplicateur $\tau$ fonction de la pente locale du site

\section{JUSTIFICATIONS VIS-A-VIS DES RISQUES ASSOCIÉS A LA LIQUÉFACTION DES SOLS}

La démarche proposée par les règles P.S. 86 pour traiter des risques associés à la liquéfaction des sols est progressive dans les moyens auxquels elle fait appel.

Paramètres d'identification des Sols

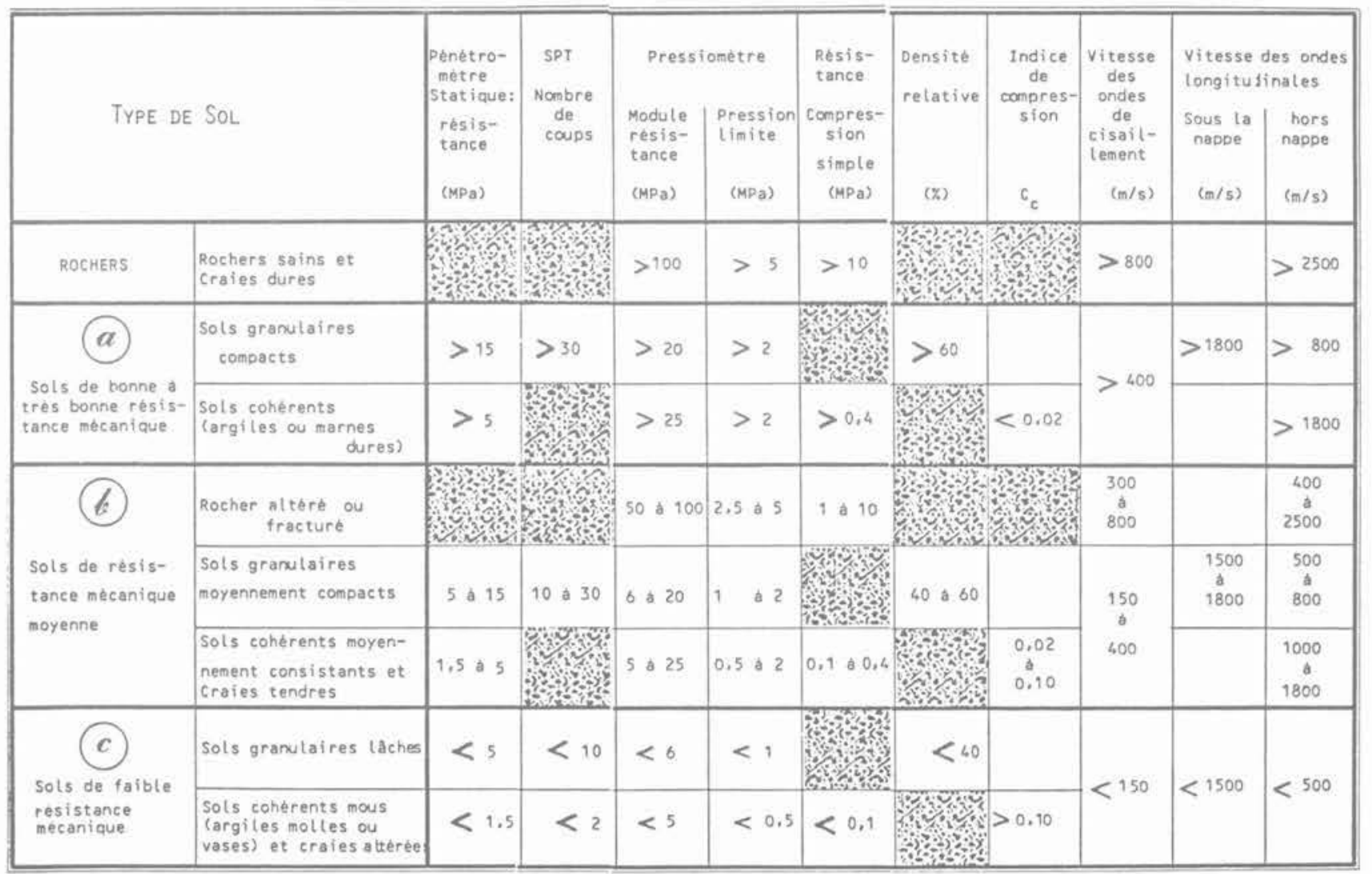

Fig. 2. - Paramètres d'identification des sols. 


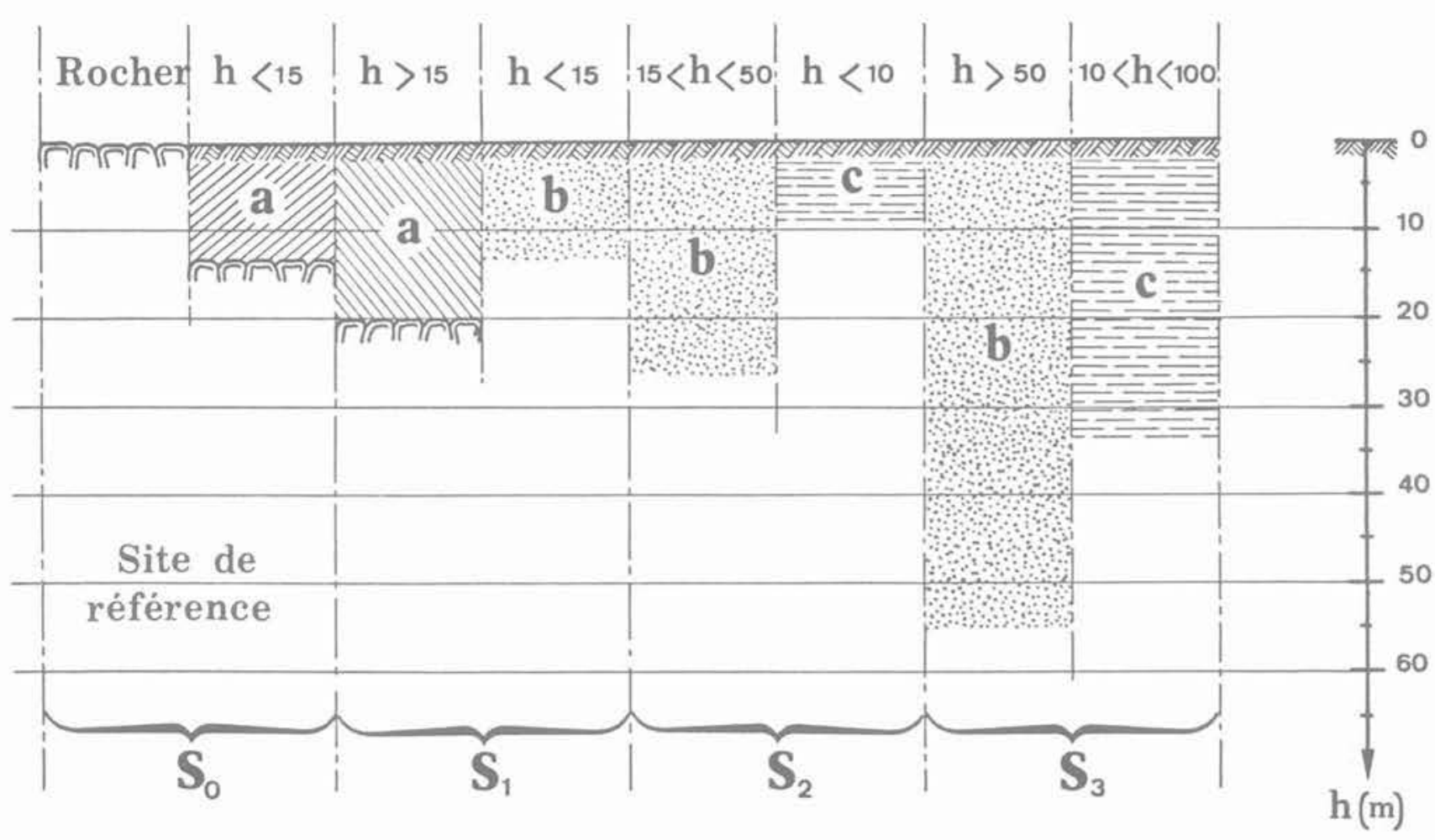

Fig. 3. - Classification des sites.

Une première série de critères associés aux résultats des essais classiques d'identification (granulométrie, limites d'Atterberg, teneur en eau), permet de reconnaitre les sols que l'on doit a priori tenir comme suspects ou non de liquéfaction. En cas de doute, il faut procéder à des investigations complémentaires.

Le diagnostic en deuxième phase peut être formulé par le biais de corrélations empiriques, considérées comme bien établies, entre les résultats de certains essais de pénétration (Standard Penetration Test, pénétromètre statique, piézocône) et les observations du comportement de différents sites.

Confirmation de ce diagnostic peut être fournie par des essais en laboratoire sur des échantillons, aussi peu remaniés que possible, chargés cycliquement.

Un horizon sera considéré comme liquéfiable si la valeur de la contrainte de cisaillement maximale engendrée par le séisme de calcul dans cette couche dépasse $75 \%$ de la contrainte de cisaillement cyclique provoquant la liquéfaction. Pour faire cette évaluation, les règles proposent pour chaque zone sismique le nombre de cycles équivalents à prendre en considération.

Si les études précédentes concluent qu'un horizon est liquéfiable pour le séisme de calcul retenu, on ne peut entreprendre de construire que dans l'une des hypothèses suivantes :

- il est clairement établi que la liquéfaction de cette couche est sans conséquence dommageable pour la construction,
- le sol est traité (densification, substitution, drainage, rabattement de nappe, ...) pour supprimer tout risque de liquéfaction ou pour rétablir la marge de sécurité donnée plus haut.

\section{JUSTIFICATION DE LA STABILITÉ DES PENTES}

Il doit être vérifié que les talus et versants, dans leur configuration définitive, restent stables sous l'action du séisme de calcul compte tenu des charges apportées par les constructions.

Pendant la durée de l'ébranlement sismique, les efforts d'inertie peuvent être simulés par l'effet d'efforts statiques équivalents et la vérification de la stabilité peut être alors menée par toute méthode de calcul classique reconnue, en tenant compte si nécessaire, de la chute des caractéristiques de résistance de certains matériaux sous l'action du séisme, et du développement de surpressions interstitielles dans certaines couches.

\section{JUSTIFICATION DES FONDATIONS}

La question de la justification du comportement des fondations était traitée de façon très sommaire dans les règles P.S. 69 qui mentionnaient pour toute règle de calcul que : " la sollicitation s'exerçant sur la fondation, ou une partie de la fondation, ne doit pas excéder $75 \%$ de la sollicitation que l'on peut considérer 
comme susceptible d'entraîner l'apparition de l'état de ruine, compte tenu, le cas échéant, des modifications des propriétés mécaniques du sol consécutives à l'action sismique».

Les règles P.S. 86 proposent cette fois non seulement des critères de stabilité vis-à-vis de la résistance du sol support mais des méthodes de calcul simplifiées applicables aux cas courants pour déterminer l'état de sollicitation dans la fondation et le sol.

\section{Les cas à considérer sont :}

- d'une part, la situation au cours du séisme avec les effets des actions dynamiques dues à la déformation propre du sol environnant, à la structure portée, et à leurs interactions;

- d'autre part, la situation après séisme en prenant en compte les effets d'éventuelles déformations rémanentes des sols d'assise.

En complément de ces vérifications numériques, les règles P.S. préconisent l'adoption d'un certain nombre de dispositions constructives pour assurer la sécurité de la construction.

\subsection{Les fondations superficielles}

Les fondations sur semelle ou radier sont à réserver aux sites constitués de sols compacts et homogènes pour lesquels l'amplitude des déformations pendant et après le séisme ne peut être préjudiciable à la bonne tenue de la construction.

Le système de fondation doit être de conception simple et homogène pour l'ensemble de l'ouvrage. Les semelles isolées établies sur sol meuble doivent être, en règle générale, liaisonnées entre elles. Lorsque la construction ne comporte pas de parties enterrées, il est conseillé de prévoir une bêche périphérique rendant solidaire l'ensemble des structures et des fondations avec le sol d'assise afin de s'opposer à leur déplacement relatif dans le plan horizontal.

La méthode et le niveau de discrétisation doivent être choisis en fonction de l'importance de linteraction entre le sol et l'ensemble de la construction. Lorsque l'on procède à une justification globale avec modélisation à la fois de la structure, des fondations et du sol, le modèle doit rendre compte du comportement non linéaire des sols.

Pour les petites constructions et lorsque l'interaction entre le sol et la structure est faible, on admet que les divers points de fondation suivent la déformée imposée au sol par l'action sismique de référence en l'absence de construction.

La réponse de la structure est alors évaluée en imposant à l'interface bâtiment-fondation le mouvement sismique de calcul correspondant au site. Les éléments de fondation sont quant à eux justifiés en tenant compte des sollicitations verticales et horizontales engendrées par la réponse de la structure au mouvement sismique.
Le comportement des fondations est vérifié vis-à-vis de l'état limite ultime de résistance du sol d'assise en s'assurant que les sollicitations sous action sismique sont au plus égales aux charges de rupture du sol divisées par 1,5.

\subsection{Les fondations profondes}

Lors d'un séisme, les éléments de fondation profonde (pieux ou barrettes) se trouvent sollicités à la fois par le déplacement du sol environnant et par la structure portée. Le niveau de sollicitation dépendra, quant à lui, des interactions s'exerçant entre le sol et les éléments de fondations, la structure portée, ses fondations et le sol sur la partie enterrée de la construction,

Il s'agit donc d'une situation très complexe qui ne peut en toute rigueur s'analyser correctement que dans un modèle global incluant le sol, les éléments de fondation et la structure. Il n'existe cependant pas actuellement de méthode de calcul d'ensemble suffisamment simple d'emploi pour être appliquée aux constructions courantes. Pour ces dernières, il était donc nécessaire de définir des méthodes de justification simplifiées pouvant être appliquées à l'aide des outils de calcul usuels et à partir des éléments d'information que l'on peut attendre d'une campagne d'investigations géotechniques à l'échelle du projet. Tout en étant simple la méthode se doit d'être d'une approche physique "saine" et conduire à un dimensionnement que l'on attend être du côté de la sécurité.

La méthode simplifiée proposée dans les règles P.S. 86 consiste à justifier séparément les éléments de fondation et la structure selon la démarche suivante:

- on considère que les pieux suivent les mouvements horizontaux imposés au sol en champ libre sans les modifier;

- la réponse de la structure est évaluée en imposant à sa base le mouvement sismique de calcul correspondant au site.

Cette méthode ne peut être appliquée que si plusieurs conditions sont satisfaites :

- la construction est suffisamment encastrée dans le sol pour qu'il n'y ait pas de déplacement relatif sensible entre la structure et le sol environnant;

- la flexibilité des éléments de fondation profonde doit être également suffisante pour que l'intégrité des éléments de fondation soit assurée sous l'hypothèse qu'ils suivent les mouvements du sol en champ libre.

La première condition permet de compter sur la participation du sol par mise en butée autour de la partie enterrée de la construction; elle explique pourquoi, dans le calcul de justification des éléments de fondation, on ne cumule pas les sollicitations horizontales venant du déplacement imposé par le sol et celles issues de la réponse inertielle de la structure (ces sollicitations ne se produisant par ailleurs pas en phase).

Cette hypothèse de non-cumul se trouve confortée par des calculs effectués a posteriori sur des constructions existantes ayant éprouvé un séisme. 
Pour le calcul des sollicitations imposées par le mouvement du sol, on admet pour déformée du profil de sol soit le résultat d'un calcul de réponse direct, soit le premier mode de vibration en champ libre.

En présence d'un profil de sol homogène ou d'un profil stratifié dans lequel les caractéristiques mécaniques varient peu d'une couche à l'autre, on pourra admettre que la déformée a l'allure d'un quart de sinusoïde. Dans ce cas, un calcul statique classique de poutre sur appuis élastiques articulée en pied et encastrée en tête, montre qu'avec les élancements courants d'éléments de fondation profonde, même dans des sols très médiocres, la déformée du pieu peut être assimilée sans écart notable à la déformée du sol.

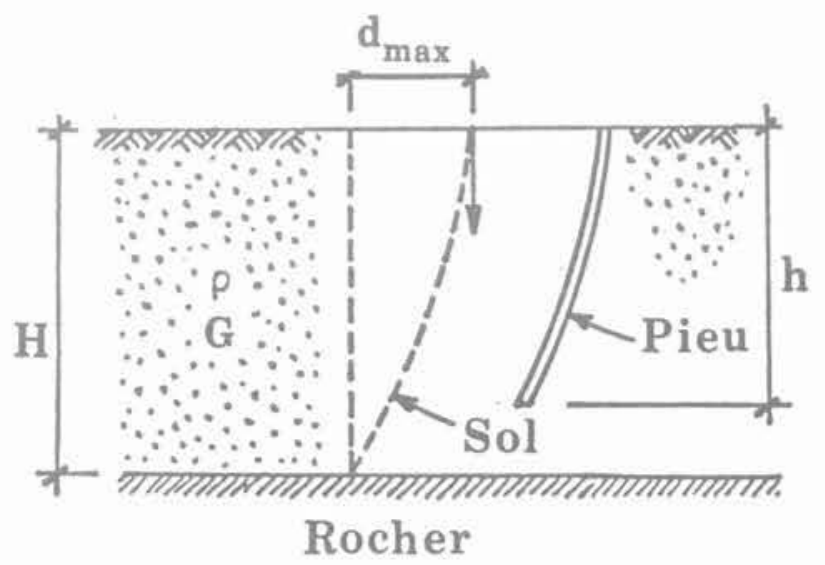

Dans les autres cas (profil de sol très hétérogène, élément de fondation très rigide), on déterminera par un calcul statique la déformée que prendrait le pieu ou la barrette si l'on impose au sol la déformée du premier mode de vibration.

La méthode proposée dans les règles P.S. 86 amène à considérer que le comportement des éléments de fondation profonde doit être justifié principalement en termes de déplacements imposés et non vis-à-vis d'efforts imposés comme peuvent l'être les éléments de la structure dans les méthodes de calcul statique équivalent. En cela, les règles P.S. 86 se démarquent nettement par leur originalité des autres codes de construction parasismique.

Le comportement des fondations est vérifié vis-à-vis de l'état limite ultime de résistance du sol au cours et après le séisme, en considérant que, pour les fondations profondes ancrées sur un substratum très résistant, les terrains de couverture ne participent pas à la reprise des efforts verticaux. Par contre, on devra tenir compte du frottement négatif si l'on suspecte un tassement possible des terrains de couverture.

Vis-à-vis de l'état limite ultime de résistance du sol, on s'assurera que les sollicitations sous action sismique sont au plus égales aux charges de rupture du sol divisées par 2 pour le terme de pointe et par 1,33 pour le frottement latéral.

Les fondations doivent être conçues et calculées de telle façon que les états ultimes de fondation ne puissent être atteints avant les états ultimes de la structure.

La présentation de méthodes de calcul est complétée par la donnée des dispositions constructives, principalement de règles de ferraillage des pieux et barrettes en béton armé. Ces dispositions sont présentées en détail dans la communication de M. R. SOULOUMIAC. 
